Mathematical Modelling AND AnAlysis

Volume 16 Number 4, December 2011, 549-557

http://dx.doi.org/10.3846/13926292.2011.628418

(c) Vilnius Gediminas Technical University, 2011
Publisher: Taylor\&Francis and VGTU

http://www.tandfonline.com/TMMA

Online ISSN: 1648-3510

Print ISSN: 1392-6292

\title{
Fault Detection in Discrete Dynamic Systems with Uncertainty Based on Interval Optimization*
}

\section{Wei Li and Xiaoli Tian}

\author{
Institute of Operational Research \& Cybernetics, Hangzhou Dianzi University \\ Hangzhou, 310018, China \\ E-mail(corresp.): weilihz@126.com
}

Received December 3, 2010; revised July 30, 2011; published online November 1, 2011

\begin{abstract}
The imprecision and the uncertainty of many systems can be expressed with interval models. This paper presents a method for fault detection in uncertain discrete dynamic systems. First, the discrete dynamic system with uncertain parameters is formulated as an interval optimization model. In this model, we also assume that there are some errors of observation values of the inputs/outputs. Then, M. Hladík's newly proposed algorithm is exploited for this model. Some numerical examples are also included to illustrate the method efficiency.
\end{abstract}

Keywords: fault detection, uncertain discrete dynamic systems, interval optimization.

AMS Subject Classification: $37 \mathrm{~N} 35 ; 37 \mathrm{~N} 40$.

\section{Introduction}

Fault detection is an issue of paramount relevance in process control from the viewpoint of improving the system reliability. The problem of fault detection in dynamic systems has drawn a lot of attention of researchers over the last two decades. In the literature such a point has been treated using several methodological frameworks, see for example [3, 5, 6, 7, 12, 23].

Whenever it is not possible to totally decouple the fault effects from the perturbation effects on the system, optimization is often used. This kind of method is based on formulation of the fault detection problem as an optimization problem. However, most of these algorithms make many idealized assumptions which are not satisfied, since in reality the system parameters may either be uncertain or time-dependent resulting in a mismatch between the real world system and the associated mathematical model $[2,14,16]$.

In this paper, a novel fault detection procedure for uncertain discrete dynamic system is proposed, based on the interval optimization technique. In

\footnotetext{
* Project partially supported by the National Natural Science Foundation of China (Grant No. 61003194, 11171316).
} 
Section 2, the problem is formulated as an optimization model. Then, Section 3 analyzes the model and M. Hladík's newly proposed algorithm is exploited for this model. Some numerical examples are given in Section 4 to illustrate the results of the method and Section 5 concludes the paper.

\section{Problem Formulation}

Consider the following discrete dynamic system

$$
y(k+n)+\sum_{i=0}^{n-1} a_{i} y(k+i)=\sum_{i=0}^{m} b_{i} u(k+i),
$$

where $a_{i}$ and $b_{i}$ are system parameters, $y$ and $u$ are observation value of inputs and outputs, respectively.

Taking the modelling errors and some other uncertain factors into account, the system parameters $a_{i}$ and $b_{i}$ may be allowed to be various in some predetermined intervals, say, $a_{i}^{L} \leqslant a_{i} \leqslant a_{i}^{R}, i=0, \ldots, n-1, b_{i}^{L} \leqslant b_{i} \leqslant b_{i}^{R}, i=0, \ldots, m$. An error will occur if some of the system parameters $a_{i}$ and $b_{i}$ are beyond the intervals $\left[a_{i}^{L}, a_{i}^{R}\right], i=0, \ldots, n-1$ or $\left[b_{i}^{L}, b_{i}^{R}\right], i=0, \ldots, m$, respectively.

Denote observation value of the inputs/outputs of the signal of the $k$ th group by

$$
\begin{aligned}
q_{k} & =[y(k+n-1), y(k+n-2), \ldots, y(k),-u(k+m), \ldots,-u(k+1),-u(k)]^{T}, \\
n_{k} & =y(k+n) .
\end{aligned}
$$

Consider $p$ groups observation of values, $k=1, \ldots, p(p>m+n)$, and denote them by

$$
Q=\left[q_{1}, \ldots, q_{p}\right]^{T}, \quad N=\left[n_{1}, \ldots, n_{p}\right]^{T} .
$$

Let $X=\left[a_{n-1}, a_{n-2}, \ldots, a_{0}, b_{m}, a_{m-1}, \ldots, b_{0}\right]^{T}$ be the system parameters. If these parameters completely match the what is actually happening, we have

$$
Q X+N=0 .
$$

Thus, we say that the observation value $[Q, N]$ and the system are consistent if there exists some system parameter

$$
X^{*}=\left[a_{n-1}^{*}, a_{n-2}^{*}, \ldots, a_{0}^{*}, b_{m}^{*}, b_{m-1}^{*}, \ldots, b_{0}^{*}\right]^{T}
$$

such that $Q X^{*}+N=0$, where $a_{i}^{*} \in\left[a_{i}^{L}, a_{i}^{R}\right], i=0, \ldots, n-1, b_{j}^{*} \in\left[b_{j}^{L}, b_{j}^{R}\right]$, $j=0, \ldots, m$. Otherwise they are inconsistent.

The consistency of the system can be verified by solving a simple linear program $Q X+N=0, X^{L} \leqslant X \leqslant X^{R}$. Nevertheless, for a reason that will be explained later, we would like to formulate the fault diagnosis for this discrete dynamic system alternatively as a convex quadratic optimization problem

$$
\begin{aligned}
& \min (Q X+N)^{T}(Q X+N) \\
& \text { s.t. } X^{L} \leqslant X \leqslant X^{R},
\end{aligned}
$$


where

$$
\begin{aligned}
& X^{L}=\left[a_{n-1}^{L}, a_{n-2}^{L}, \ldots, a_{0}^{L}, b_{m}^{L}, b_{m-1}^{L}, \ldots, b_{0}^{L}\right]^{T}, \\
& X^{R}=\left[a_{n-1}^{R}, a_{n-2}^{R}, \ldots, a_{0}^{R}, b_{m}^{R}, b_{m-1}^{R}, \ldots, b_{0}^{R}\right]^{T} .
\end{aligned}
$$

Define the function $f(X)=(Q X+N)^{T}(Q X+N)$. Thus, the observation value and the system are consistent if there exists $X^{*} \in\left[X^{L}, X^{R}\right]$ such that $f\left(X^{*}\right)=0$, or $\left|f\left(X^{*}\right)\right| \leqslant \varepsilon$, where $\varepsilon$ is a predetermined tolerance. Otherwise they are inconsistent. Here the optimal value of the problem (2.3) is used as the threshold value. Clearly, model (2.3) is equivalent to the model

$$
\begin{aligned}
& \min \frac{1}{2} X^{T} Q^{T} Q X+N^{T} Q X \\
& \text { s.t. } X^{L} \leqslant X \leqslant X^{R}
\end{aligned}
$$

since

$$
(Q X+N)^{T}(Q X+N)=2\left(\frac{1}{2} X^{T} Q^{T} Q X+N^{T} Q X\right)+N^{T} N .
$$

Further, let

$$
H=Q^{T} Q, \quad Y=X-X^{L}, \quad C^{T}=\left(X^{L}\right)^{T} H+N^{T} Q .
$$

The model (2.4) becomes

$$
\begin{aligned}
& \min \frac{1}{2} Y^{T} H Y+C^{T} Y \\
& \text { s.t. } Y \geqslant 0, Y \leqslant X^{R}-X^{L} .
\end{aligned}
$$

For universality, we assume that the system parameter vector $Y$ is to be a subject to a more general linear relation $A Y \leqslant B$, under which the system is consistent. This linear relation can be determined according to the characteristic of the system considered. Thus, the model (2.4) is translated into a more general model below

$$
\begin{aligned}
& \min \frac{1}{2} Y^{T} H Y+C^{T} Y \\
& \text { s.t. } Y \geqslant 0, A Y \leqslant B,
\end{aligned}
$$

where

$$
\frac{1}{2} Y^{T} H Y+C^{T} Y=\left(\frac{1}{2} X^{T} Q^{T} Q X+N^{T} Q X\right)-\left(\frac{1}{2} X^{L T} Q^{T} Q X^{L}+N^{T} Q X^{L}\right)
$$

Model (2.7) is a standard convex quadratic optimization problem, which can be solved in a finite number of steps.

In reality however, not only the system parameters, but also observation values of the inputs/outputs of the signal vary in some interval. We say that such discrete dynamic systems are uncertain discrete dynamic systems. In this 
paper, we first formulate the uncertain discrete dynamic systems as an interval optimization problem. Then one effective solution algorithm is developed.

For simplicity, some notations are first introduced. Superscript, I, on a quantity indicates that the quantity is an interval (number, vector, or matrix). Quantities without a superscript are real (numbers, vectors, or matrices). The left endpoint of an interval is indicated by a superscript, $L$, and the right endpoint by a superscript, $R$. Thus a scalar interval $a^{I}$ is given by $\left[a^{L}, a^{R}\right]$. The vector of left endpoints of an interval vector, $x^{I}$, is denoted by $x^{L}$; and the vector of right endpoints by $x^{R}$. Thus, we write an interval vector, $x^{I}$, as $\left[x^{L}, x^{R}\right]$. Similarly, we write an interval matrix, $A^{I}$, as $\left[A^{L}, A^{R}\right]$.

We say that a real vector $x \in R^{n}$ is contained in an interval vector $x^{I}$ and write $x \in x^{I}$, if $x_{i}^{L} \leqslant x_{i} \leqslant x_{i}^{R}$ for all $i=1, \ldots, n$. We say that a real matrix $A \in R^{m \times n}$ is contained in an interval matrix $A^{I}$ and write $A \in A^{I}$, if $a_{i j}^{L} \leqslant a_{i j} \leqslant a_{i j}^{R}$ for all $i=1, \ldots, m, j=1, \ldots, n$.

Now assume that the system parameters lie in some allowable interval and the observation value of the inputs/outputs of the signal varies in some error interval. Thus the matrices $Q, N$ in (2.1) are changed into interval matrices $Q^{I}=\left[q_{1}^{I}, \ldots, q_{p}^{I}\right]^{T}, N^{I}=\left[n_{1}^{I}, \ldots, n_{p}^{I}\right]^{T}$. The interval counterpart to $(2.2)$ is

$$
Q^{I} X+N^{I}=0, \quad X^{L} \leqslant X \leqslant X^{R},
$$

which is an interval linear system.

It is well known that the optimal problems with constraints of the interval system $A^{I} x=b^{I}, x \geqslant 0$ are more difficult than that with the system $A^{I} x \leqslant b^{I}$, $x \geqslant 0$ (cf. [10,4]). Thus, we would like to formulate the fault diagnosis for this discrete dynamic system with inexact inputs/outputs alternatively as an interval convex quadratic optimization model.

In a way similar to the analysis presented above, we obtain the interval convex quadratic optimization model for uncertain discrete dynamic system as follows

$$
\begin{aligned}
& \min \frac{1}{2} Y^{T} H^{I} Y+\left(C^{I}\right)^{T} Y \\
& \text { s.t. } Y \geqslant 0, A^{I} Y \leqslant B^{I},
\end{aligned}
$$

where the system parameter restriction is assumed to be subject to general linear relation $A^{I} Y \leqslant B^{I}$, under which the system is consistent. This general form makes the model more generally or universally applicable.

In recent years, many papers were devoted to study the solvability of the interval linear systems, see e.g. $[1,17,18,19,20,21]$. Rohn gave many complexity results related to interval problems, and proved their NP-hardness (see $[18,19,20,21])$. His papers provide a deep insight into the algebraic properties of linear interval systems. However, in this paper, we would like to use the interval model (2.10) instead of model (2.9), in order to exploit algorithm, which is recently proposed by Hladík (see, Hladík, 2011 [11]). The interval convex quadratic optimization problem is studied by some authors, see, e.g., $[11,13]$.

From the discussions given above, we know that the interval arithmetic is required for the formulation and the solution of the problem (2.10). A complete 
discussion on interval arithmetic can be found in $[1,17]$. Due to the well known dependency problem, the problem (2.10) is not equivalent to the original system, but for narrow intervals it can yield a sufficiently tight approximation. The detailed discussions on dependency can be found in $[1,8,9,10,15,17]$.

\section{Problem Solving}

\subsection{Problem analysis}

The problem (2.10) can be also described as

$$
\begin{aligned}
& \min \frac{1}{2} Y^{T} H Y+C^{T} Y \\
& \text { s.t. } Y \geqslant 0, A Y \leqslant B,
\end{aligned}
$$

where $H, A, C$ and $B$ vary in given interval matrices $H^{I}, A^{I}$ and interval vectors $C^{I}, B^{I}$. An optimal solution $Y^{*}$ to the problem (3.1) is defined as an optimal solution to some scenario. That is, for some $A \in A^{I}, B \in B^{I}, c \in c^{I}$, $H \in H^{I}, Y^{*}$ is an optimal solution to the problem (3.1).

Denote by $\left[f^{L}, f^{R}\right]$ the range in which the optimal value of (3.1) varies. From (2.5) and (2.8) we know that

$$
\left(Q^{I} X+N^{I}\right)^{T}\left(Q^{I} X+N^{I}\right)=2\left(\frac{1}{2} Y^{T} H^{I} Y+\left(C^{I}\right)^{T} Y\right)+\omega^{I}
$$

where

$$
\omega^{I}=\left(N^{I}\right)^{T} N^{I}+2\left(N^{I}\right)^{T} Q^{I} X^{L}+X^{L^{T}}\left(Q^{I}\right)^{T} Q^{I} X^{L}
$$

is an interval number. Thus, the threshold interval is obtained by

$$
\left[2 f^{L}, 2 f^{R}\right]+\omega^{I}
$$

once the range of optimal value range $\left[f^{L}, f^{R}\right]$ is available. The system is consistent if and only if $\left|\left[2 f^{L}, 2 f^{R}\right]+\omega^{I}\right|<\varepsilon$, where $\varepsilon$ is a predetermined threshold according to the characteristic of the system considered, and $\left|a^{I}\right|$ denotes the length of the interval number $a^{I}$. In general, we may assume that a normalized threshold $\varepsilon$ lies in the interval $(0,1)$. In reality, $\varepsilon$ is chosen according to the characteristic of the system considered and the demand of the decision maker. In examples of Section 4 , we use 0.5 , the middle value of the interval $(0,1)$.

In the next subsection, we provide Hladík's newly proposed solution method [11] for interval convex quadratic programming, by which the threshold interval can be easily obtained.

\subsection{Hladík's solution method for interval convex quadratic pro- gramming}

The objective function value of the fault diagnosis model (2.10) (or (3.1)) lies in an interval number $\left[f^{L}, f^{R}\right]$. Clearly, the lower and the upper bound of 
the objective function value of the model (2.10) (or (3.1)), $f^{L}$ and $f^{R}$, can be described as interval convex quadratic programming models:

$$
\begin{aligned}
& f^{L}=\min _{H \in H^{I}, C \in C^{I}, A \in A^{I}, B \in B^{I}} \min _{Y} \frac{1}{2} Y^{T} H Y+C^{T} Y \\
& \text { s.t. } Y \geqslant 0, A Y \leqslant B
\end{aligned}
$$

and

$$
\begin{aligned}
& f^{R}=\max _{H \in H^{I}, C \in C^{I}, A \in A^{I}, B \in B^{I}} \min _{Y} \frac{1}{2} Y^{T} H Y+C^{T} Y \\
& \text { s.t. } Y \geqslant 0, A Y \leqslant B
\end{aligned}
$$

respectively. To obtain the lower bound $f^{L}$ and upper bound $f^{R}$, we should determine the optimal solution of model (3.4) and (3.5). Some results for these cases were developed by X.Y. Wu, G.H. Huang, L. Liu, J.B.Li (2006) [22] and by Liu and Wang (2007) [13], respectively. An algorithm for this model is also proposed in the original manuscript of this paper. However, during the process of revising this paper, we have learned of M. Hladík's solution method for interval nonlinear programming, based on dual theory of interval programming [11]. As a special case, M. Hladík obtained the lower bound $f^{L}$ and upper bound $f^{R}$ of interval convex quadratic program by two simple non-interval quadratic programming problems. Thus, our original solution method can be greatly simplified by using this new solution method, described below.

Proposition 1 [Hladík, 2011]. We have

$$
\begin{array}{ll}
f^{L}=\min \frac{1}{2} Y^{T} H^{L} Y+C^{L} Y & f^{R}=\min \frac{1}{2} Y^{T} H^{R} Y+C^{R T} Y \\
\text { s.t. } Y \geqslant 0, A^{L} Y \leqslant B^{R}, & \text { s.t. } Y \geqslant 0, A^{R} Y \leqslant B^{L} .
\end{array}
$$

Once the lower bound $f^{L}$ and upper bound $f^{R}$ of the problem (2.10) (or (3.1)) are obtained, the threshold interval (3.3) is given by

$$
\left[2 f^{L}+\underline{\omega}, 2 f^{R}+\bar{\omega}\right] .
$$

\section{Illustrative Examples and Remarks}

In this section, two numerical examples are discussed to demonstrate the proposed method.

Example 1. Assume that the system parameter vector $X$ varies in the following interval $X=([-1,1],[0,2])^{T}$, in which case the system works well. Assume that the observation value of the inputs/outputs of the signal varies in the matrices

$$
Q=\left(\begin{array}{cc}
{[1,2]} & 1 \\
1 & {[2,3]}
\end{array}\right), \quad N=\left(\begin{array}{l}
{[1,2]} \\
{[2,3]}
\end{array}\right)
$$


respectively. Denote $Y$ by $Y=X-X^{L}$, the system parameter restriction is assumed to be subject to linear relation $A^{I} Y \leqslant B^{I}$, where

$$
A=\left(\begin{array}{cc}
{[4,8]} & 1 \\
{[1,2]} & {[-8,-4]}
\end{array}\right), \quad B=\left(\begin{array}{l}
2 \\
2
\end{array}\right) .
$$

Given the threshold $\varepsilon=0.5$. The fault diagnosis model of this uncertain discrete dynamic systems, according to (2.4), (2.6) and (2.10), can be formulated as

$$
\begin{array}{ll}
\min [-2,5] Y_{1}+[0,8] Y_{2}+[1,2.5] Y_{1}^{2}+[3,5] Y_{1} Y_{2}+[2.5,5] Y_{2}^{2} \\
\text { s.t. } \quad[4,8] Y_{1}+Y_{2} \leqslant 2, \\
\quad[1,2] Y_{1}+[-8,-4] Y_{2} \leqslant 2, \quad Y_{1}, Y_{2} \geqslant 0 .
\end{array}
$$

Using (3.6), $f^{L}$ and $f^{R}$ can be determined respectively by

$$
\begin{aligned}
& f^{L}=\min -2 Y_{1}+Y_{1}^{2}+3 Y_{1} Y_{2}+2.5 Y_{2}^{2} \\
& \text { s.t. } \quad 4 Y_{1}+Y_{2} \leqslant 2, \quad Y_{1}-8 Y_{2} \leqslant 2, \quad Y_{1}, Y_{2} \geqslant 0 .
\end{aligned}
$$

and

$$
\begin{aligned}
& f^{R}=\min 5 Y_{1}+8 Y_{2}+2.5 Y_{1}^{2}+5 Y_{1} Y_{2}+5 Y_{2}^{2}, \\
& \text { s.t. } \quad 8 Y_{1}+Y_{2} \leqslant 2, \quad 2 Y_{1}-4 Y_{2} \leqslant 2, \quad Y_{1}, Y_{2} \geqslant 0 .
\end{aligned}
$$

By employing the function quadprog in Matalab 6.5, we derive the upper bound $f^{R}=0$ and the lower bound $f^{L}=-0.75$. Then, using (3.2) and (3.7) we obtain the threshold interval $[-8.5,12]$. The length of this interval is $12+8.5>0.5$, we say that the same error occur with the system.

Example 2. Assume that the allowable interval of the system parameter vector $X$ is $X=([0,1],[0.5,2])^{T}$. Assume that the observation value of the inputs/outputs of the signal varies in the matrices

$$
Q=\left(\begin{array}{cc}
{[0.125,0.25]} & 0.125 \\
0.125 & {[0.25,0.375]}
\end{array}\right), \quad N=\left(\begin{array}{l}
{[0.125,0.25]} \\
{[0.25,0.375]}
\end{array}\right)
$$

respectively. Denote $Y$ by $Y=X-X^{L}$, the system parameter restriction is assumed to be subject to linear relation $A^{I} Y \leqslant B^{I}$, where

$$
A=\left(\begin{array}{cc}
{[4,8]} & 1 \\
{[1,2]} & {[-8,-4]}
\end{array}\right), \quad B=\left(\begin{array}{l}
2 \\
2
\end{array}\right) .
$$

Given the threshold $\varepsilon=0.5$. The fault diagnosis model of these uncertain discrete dynamic systems, according to (2.4), (2.6) and (2.10), can be formulated as

$$
\begin{array}{cc}
\min & {[0.0703,0.1484] Y_{1}+[0.1172,0.25] Y_{2}+[0.0157,0.0391] Y_{1}^{2}} \\
& +[0.0469,0.0781] Y_{1} Y_{2}+[0.0391,0.0782] Y_{2}^{2} \\
\text { s.t. } \quad & {[4,8] Y_{1}+Y_{2} \leqslant 2,} \\
& {[1,2] Y_{1}+[-8,-4] Y_{2} \leqslant 2, \quad Y_{1}, Y_{2} \geqslant 0 .}
\end{array}
$$

Then we have

$$
\begin{aligned}
& f^{L}=\min 0.0703 Y_{1}+0.1172 Y_{2}+0.0157 Y_{1}^{2}+0.0469 Y_{1} Y_{2}+0.0391 Y_{2}^{2} \\
& \text { s.t. } \quad 4 Y_{1}+Y_{2} \leqslant 2, \quad Y_{1}-8 Y_{2} \leqslant 2, \quad Y_{1}, Y_{2} \geqslant 0
\end{aligned}
$$


and

$$
\begin{aligned}
& f^{R}=\min 0.1484 Y_{1}+0.25 Y_{2}+0.0391 Y_{1}^{2}+0.0781 Y_{1} Y_{2}+0.0782 Y_{2}^{2}, \\
& \text { s.t. } \quad 8 Y_{1}+Y_{2} \leqslant 2, \quad 2 Y_{1}-4 Y_{2} \leqslant 2, \quad Y_{1}, Y_{2} \geqslant 0 .
\end{aligned}
$$

By employing the function quadprog in Matalab 6.5, we derive the upper bound $f^{R}=0$ and the lower bound $f^{L}=0$. Then, using (3.2) and (3.7) we obtain the threshold interval $[0.1758,0.4141]$. The length of this interval is $0.4141-0.1785=0.2356<0.5$, we say that the the system is consistent.

\section{Conclusion}

In this paper, a method for fault detection in uncertain discrete dynamic systems is proposed. The key feature of the method is the consideration of both system parameter uncertainties in the model of the system and the objective errors of the observation value of the inputs/outputs. An interval optimization model is formulated to describe not only the disturbances caused by unknown inputs, but also by uncertainties of parameters. This model can be handled effectively by translating it to two classical quadratic optimization problems, based on the duality theory of the optimization. As mentioned in the end of the Section 2, the program (2.10) is an approximation of the original system, due to the phenomenon of dependency. It is interesting to study the effect of overestimation deeply, and to investigate quality of the approximation.

\section{Acknowledgements}

The authors acknowledge the editor and the referees for their helpful corrections and suggestions, which have improved the quality of the paper. We also thank Prof. M. Hladík for providing us the references [10, 11].

\section{References}

[1] G. Alefeld and J. Herzberger. Introduction to Interval Computations. Academic Press, London, 1983.

[2] A. Casavola, D. Famularo and G. Franzé. Robust fault detection of uncertain linear systems via quasi-LMIs. Automatica, 44:289-295, 2008. http://dx.doi.org/10.1016/j.automatica.2007.05.010.

[3] J. Chen and R. Patton. Robust Model-Based Fault Diagnosis for Dynamic Systems. Kluwer Academic Publishers, Norwell, MA, USA, 1999.

[4] M. Fiedler, J. Nedoma, J. Ramík, J. Rohn and K. Zimmermann. Linear Optimization Problems with Inexact Data. Springer, New York, 2006.

[5] P.M. Frank and X. Ding. Frequency domain approach to optimally robust residual generation and evaluation for model-based fault diagnosis. Automatica, 30:789-804, 1994. http://dx.doi.org/10.1016/0005-1098(94)90169-4.

[6] P.M. Frank and X. Ding. Survey of robust residual generation and evaluation methods in observer-based fault detection systems. Journal of Process Control, 7:403-424, 1997. http://dx.doi.org/10.1016/S0959-1524(97)00016-4. 
[7] P.M. Frank, E.A. García and B. Köppen-Seliger. Modelling for fault detection and isolation versus modelling for control. Math. Comput. Simulation, 53:259271, 2000. http://dx.doi.org/10.1016/S0378-4754(00)00212-3.

[8] M. Hladík. Description of symmetric and skew-symmetric solution set. SIAM J. Matrix Anal. Appl., 30(2):509-521, 2008. http://dx.doi.org/10.1137/070680783.

[9] M. Hladík. Optimal value range in interval linear programming. Fuzzy Optim. Decis. Mak., 8(3):283-294, 2009. http://dx.doi.org/10.1007/s10700-009-9060-7.

[10] M. Hladík. Interval linear programming: A survey. In Zoltan Adam Mann(Ed.), Linear Programming - New Frontiers, pp. 1-46. Nova Science Publishers, Inc., 2011.

[11] M. Hladík. Optimal value bounds in nonlinear programming with interval data. TOP. in press, 2011. http://dx.doi.org/10.1007/s11750-009-0099-y.

[12] J. Liu, J.L. Wang and G.H. Yang. An LMI approach to minimum sensitivity analysis with application to fault detection. Automatica, 41:1995-2004, 2005. http://dx.doi.org/10.1016/j.automatica.2005.06.005.

[13] S.T. Liu and R.T. Wang. A numerical solution method to interval quadratic programming. Appl. Math. Comput., 189(2):1274-1281, 2007. http://dx.doi.org/10.1016/j.amc.2006.12.007.

[14] B. Möller, W. Graf, J.U. Sickert and U. Reuter. Numerical simulation based on fuzzy stochastic analysis. Math. Comput. Model. Dyn. Syst., 13:349-364, 2007. http://dx.doi.org/10.1080/13873950600994514.

[15] R.E. Moore. Interval Analysis. Prentice-Hall, New Jersey, 1966.

[16] S.A. Nazin and B.T. Polyak. Interval parameter estimation under model uncertainty. Math. Comput. Model. Dyn. Syst., 11:225-237, 2005. http://dx.doi.org/10.1080/138950500069243.

[17] A. Neumaier. Interval Methods for Systems of Equations. Cambridge University Press, Cambridge, 1990.

[18] J. Rohn. NP-Hardness Results for Some Linear and Quadratic Problems. Institute of Computer Science, Academy of Sciences of the Czech Republic, Prague, 1995.

[19] J. Rohn. Linear programming with inexact data is NP-hard. ZAMM, Z. Angew. Math. Mech., 78(Suppl. 3):1051-1052, 1998. http://dx.doi.org/10.1002/zamm.19980781594.

[20] J. Rohn. Solvability of systems of linear interval equations. SIAM J. Matrix Anal. Appl., 25:237-245, 2003. http://dx.doi.org/10.1137/S0895479801398955.

[21] J. Rohn. Solvability of systems of interval linear equations and inequalities. In M. Fiedler, J. Nedoma, J. Ramík, J. Rohn and K. Zimmermann(Eds.), Linear Optimization Problems with Inexact Data, chapter 2, pp. 35-77. Springer, New York, 2006.

[22] X.Y. Wu, G.H. Huang, L. Liu and J.B. Li. An interval nonlinear program for the planning of waste management systems with economies-of-scale effects - A case study for the region of Hamilton, Ontario, Canada. European J. Oper. Res., 171(2):349-372, 2006. http://dx.doi.org/10.1016/j.ejor.2005.01.038.

[23] E. Zahariev and J. Mcphee. Stabilization of multiple constraints in multibody dynamics using optimization and a pseudo-inverse matrix. Math. Comput. Model. Dyn. Syst., 9:417-435, 2003. http://dx.doi.org/10.1076/mcmd.9.4.417.27898. 